T. KAWACHI

KODAI MATH. J.

21 (1998), 185-191

\title{
ON $d$-SPANNEDNESS OF THE ADJOINT BUNDLES ON POLARIZED MANIFOLDS
}

\author{
TAKESHI KAWACHI
}

\section{Introduction}

Let $X$ be a projective variety and $L$ be an ample Cartier divisor. Then the pair $(X, L)$ is called a polarized variety.

Let $K$ be an algebraically closed field of characteristic $p \geq 0$. Let $X$ be an $n$-dimensional smooth variety over $K$. Denote by $X^{[r]}$ the Hilbert scheme of all zero dimensional subschemes $\left(Z, \mathcal{O}_{Z}\right)$ of $X$ with length $\left(\mathcal{O}_{Z}\right)=r$. An element of $X^{[r]}$ is sometimes called a zero cycle.

Denition ([BB (0.0)]). An element $\left(Z, \mathcal{O}_{Z}\right)$ of $X^{[r]}$ is called curvilinear if $\operatorname{dim} T_{v} Z \leq 1$ for every $v \in Z_{\text {red }}$.

Denition ([BB (0.0)]). Let $L$ be a line bundle on $X$ and let $d \geq 0$. We say that $L$ is $d$-spanned if $\Gamma(L) \rightarrow \Gamma\left(\mathcal{O}_{Z}(L)\right)$ is surjective for any curvilinear zero cycle $Z \in X^{[d+1]}$.

Note that $L$ is 0 -spanned if and only if $L$ is generated by global sections. Note also that $L$ is 1 -spanned if and only if $L$ is very ample.

Note that if $L$ is $d$-spanned and $\left(Z, \mathcal{O}_{Z}\right)$ is a curvilinear zero cycle with length $\left(\mathcal{O}_{Z}\right)=m \leq d+1$, then the linear span $\langle Z\rangle$ by $H^{0}(X, L)$ is isomorphic to $\boldsymbol{P}^{m-1}$.

On the $d$-spannedness, the following Fujita's conjecture is well known.

CONJECTURE $([\mathrm{F} 1])$. Let $(X, L)$ an smooth polarized $n$-dimensional variety over $C$. If $L^{n}>1$ then

(1) $K_{X}+t L$ is spanned for $t \geq n$.

(2) $K_{X}+t L$ is very ample for $t \geq n+1$.

This conjecture is true if $n \leq 2$ by [R]. The case (1) of above conjecture is also true if $n \leq 4$ (by [EL] and [F2] if $n=3$ and by [K] if $n=4$ ). If $n=2$, the following theorem holds.

1991 Mathematics Subject Classification. Primary 14F05, Secondary 14C20, 14 J40.

Key words and phrases. polarized variety, $d$-spannedness, Fujita conjecture.

Received October 27, 1997; revised May 8, 1998. 
THEOREM ([BFS Theorem (2.2)]). Let $(S, L)$ be a polarized surface and let $d \geq 0$. If $L^{2}>1$, then $K_{S}+t L$ is $d$-spanned for any $t \geq d+2$.

When $L^{n}=1$, there are some examples where $K_{X}+n L$ is not spanned, such as $\left(\boldsymbol{P}^{n}, \mathcal{O}(1)\right)$, a hypersurface $M$ of degree 6 in the weighted projective space $\boldsymbol{P}(3,2,1, \ldots, 1)$ with $\mathcal{O}_{M}(1)$, and so on. We construct new series of such examples for $d$-spannedness.

The main result is the following:

MAIN RESULT. For any integers $d \geq 0, n \geq 2$, and for any algebraically closed field $K$, there exists an n-dimensional polarized manifold $(X, L)$ of general type with $L^{n}=1$ over $K$ such that $K_{X}+(n+d) L$ is not d-spanned.

\section{Preliminaries}

Let $q \geq 5$ be a prime number such that $q \neq p$. Let $G \cong Z / q Z$ be a cyclic group of order $q$ generated by the primitive $q$-th root of unity. Given a nonnegative integer $n$ and a sequence $w_{0}, \ldots, w_{n+1}$ with $0 \leq w_{0}<\cdots<w_{n+1} \leq q-1$, we define an action of $G$ on $\boldsymbol{P}_{K}^{n+1}$ by

$$
g \cdot\left(z_{0}: \cdots: z_{n+1}\right)=\left(g^{w_{0}} z_{0}: \cdots: g^{w_{n+1}} z_{n+1}\right)
$$

for $g \in G$.

Denote by $S_{d}^{J}$ the set of monomials $\left\{z_{l_{1}}, \ldots, z_{l_{d}}\right\}$ of degree $d$ such that $w_{i_{1}}+\cdots+w_{i_{d}} \equiv j$. Here and throughout this paper, $\equiv$ means conjugate modulo $q$, i.e. $=$ in $\boldsymbol{Z} / q \boldsymbol{Z}=\boldsymbol{F}_{q}$. Let $X$ be a smooth hypersurface in $\boldsymbol{P}_{K}^{n+1}$ defined by a $G$-invariant homogeneous polynomial $F$ of degree $q$. Let

$$
F=\sum_{f_{i} \in S_{q}^{0}} \alpha_{i} f_{i}=\alpha_{0} z_{0}^{q}+\cdots+\alpha_{n+1} z_{n+1}^{q}+\cdots=0 \quad \text { for } \alpha_{i} \in K
$$

and we assume $\alpha_{j} \neq 0$ for all $j=0, \ldots, n+1$.

Note that $F$ does not contain monomials of the form $z_{a}^{l} z_{b}^{q-\imath}, a \neq b$ and $i \neq 0$. Indeed, otherwise, $\left(w_{a}-w_{b}\right) i \equiv 0$. Since $w_{a} \not \equiv w_{b}$, this implies $i \equiv 0$.

LEMma 1. This action of $G$ on $X$ is fixed point free. Therefore the quotient space $M=X / G$ is smooth.

Proof. Assume that $g x=x$ for $x=\left(x_{0}: \cdots: x_{n+1}\right) \in X$ and for $g \neq 1$. Take $j$ such that $x_{j} \neq 0$. Since $g x=\left(g^{w_{0}} x_{0}: \cdots: g^{w_{n+1}} x_{q-1}\right)=x$, we have $\left(g^{w_{i}-w_{j}}-1\right) x_{l}=0$ for any $i \neq j$. Since the order of $g$ is $q, g^{w_{i}-w_{j}} \neq 1$ for $i \neq j$. Hence we have $x_{l}=0$ for $i \neq j$. Therefore $x=(0: \cdots: 0: 1: 0: \cdots: 0)$ but this point is not on $X$ since $\alpha_{j} \neq 0$.

Let $\pi: X \rightarrow M$ be the natural morphism which is a covering of degree $q$. 
For $a \neq b$, let $x_{a, b}$ be a point on $X$ defined by $z_{j}=0$ for all $j \neq a, b$. Such a point satisfies the equation $\alpha_{a} z_{a}^{q}+\alpha_{b} z_{b}^{q}=0$, hence is unique up to the $G$-action, and defines a unique point on $M$, which will be denoted by $y_{a, b}$.

\section{The fundamental case}

In this section we assume $n=q-2$. Let $X$ and $F=\alpha_{0} z_{0}^{q}+\cdots+$ $\alpha_{n+1} z_{n+1}^{q}+\cdots$ be as before. We assume that $\alpha_{0}, \ldots, \alpha_{n+1} \neq 0$ and $X$ is smooth.

Set $F_{i}=\left(\partial / \partial z_{l}\right) F$. Since $X$ is smooth, $F_{0}(x)=\cdots=F_{q-1}(x)=0$ does not occur at any $x \in X$.

Proposition 2. The canonical sheaf of $M=X / G$ is trivial.

Proof. The natural surjective morphism $\pi: X \rightarrow M$ is the étale Galois covering. Since $H^{l}\left(\boldsymbol{P}^{n+1}, \mathcal{O}_{\boldsymbol{P}^{n+1}}(-q)\right)=0$ for $1 \leq i \leq n$, we have

$$
H^{l}\left(X, \mathcal{O}_{X}\right)=0 \quad \text { for } 1 \leq i \leq n-1 .
$$

Since $\pi$ is finite morphism, we have $H^{\imath}\left(M, \pi_{*} \mathcal{O}_{X}\right)=H^{\imath}\left(X, \mathcal{O}_{X}\right)=0$ for $1 \leq i \leq$ $n-1$. Hence we have

$$
H^{l}\left(M, \mathcal{O}_{M}\right)=0 \quad \text { for } 1 \leq i \leq n-1,
$$

because of $\mathcal{O}_{M}$ is a component of direct sum of $\pi_{*} \mathcal{O}_{X}$. By the adjunction formula we have $\omega_{X} \cong \mathcal{O}_{X}$. Hence we have

$$
H^{n}\left(X, \mathcal{O}_{X}\right) \cong H^{0}\left(X, \omega_{X}\right)=H^{0}\left(X, \mathcal{O}_{X}\right)=K,
$$

by the Serre duality. Since $n=q-2$ is an odd number, we have $\chi\left(\mathcal{O}_{X}\right)=0$ by (1) and (3). Hence we have

$$
\chi\left(\mathcal{O}_{M}\right)=(1 / \operatorname{deg} \pi) \chi\left(\mathcal{O}_{X}\right)=0 .
$$

Since (1) and (4), we have

$$
h^{0}\left(M, \omega_{M}\right)=h^{n}\left(M, \mathcal{O}_{M}\right)=h^{0}\left(M, \mathcal{O}_{M}\right)=1
$$

Since $\omega_{X} \cong \mathcal{O}_{X}$, hence $\omega_{M}$ is numerically trivial as an element of $\operatorname{Pic}(M)$. Since (5), we have $\omega_{M} \cong \mathcal{O}_{M}$.

Let $H_{J}$ be the divisor on $X$ defined by $\left(z_{J}=0\right)$. Then $G$ acts on $H_{J}$ freely. Hence $D_{j}=H_{j} / G$ is an ample divisor on $M$. Since $H_{j}^{n}=q$ and $\pi^{*} D_{j}=H_{J}$, we have $D_{J}^{n}=1$.

Let $N=D_{1}-D_{0}$ and $N^{\prime}=D_{j}-D_{j-1}$. Since

$$
\operatorname{div}\left(\frac{z_{J-1} z_{1}}{z_{j} z_{0}}\right)=H_{J}-H_{J-1}-H_{1}+H_{0}
$$


and $f=z_{J-1} z_{1} /\left(z_{j} z_{0}\right)$ is $G$-invariant, thus $f \in K(M)$ and $\operatorname{div}(f)=N-N^{\prime}$. Therefore $N^{\prime}$ and $N$ are linearly equivalent. Hence we have $D_{i}-D_{j}=(i-j) N$ in $\operatorname{Pic}(M)$. Moreover $\mathcal{O}_{M}(N)$ is a $q$-torsion element in $\operatorname{Pic}(M)$.

Let $E=\sum_{l=0}^{q-1} a_{i} D_{i}+j N$ be a divisor on $M$ where each $a_{i} \geq 0$ and $j \geq 0$. Since $D_{i}-D_{0}=i N$, we can write $E=t D_{0}+j^{\prime} N$ where $t=\sum_{l=0}^{q-1} a_{i}$ and $j^{\prime} \geq 0$. We consider divisors of the form $t D+j N$, where $D=D_{0}$.

THEOREM 3. Let $M$ be as before.

(1) $\left|K_{M}+n D+j N\right|$ has finite base points. They are exactly the $y_{a, b}$ 's such that $a+b+j \equiv 0$.

(2) $K_{M}+(n+d) D+j N$ is not $d$-spanned for $d \leq q-1$.

Proof. For simplicity, denote $H_{0}$ by $H$. Recall that $K_{M}=0$ in this case.

(1) Since

$$
\pi^{*}: H^{0}(M, n D+j N) \rightarrow H^{0}(X, n H)
$$

is injective, we identify $H^{0}(M, n D+j N)$ with its image of $\pi^{*}$. Then $S_{n}^{j}$ can be viewed as a basis of $H^{0}(M, n D+j N)$.

Let $a \neq b$. Take a point $x_{a, b}$ in $\pi^{-1}\left(y_{a, b}\right)$. Assume that $a+b \equiv-j$. We will show that $y_{a, b}$ is a base point of $\left|K_{M}+n D+j N\right|$. If not, there exists $f \in S_{n}^{J}$ such that $f\left(x_{a, b}\right) \neq 0$. Since $z_{\iota}=0$ for $i \neq a, b$ at $x_{a, b}, f$ must be of the form $f_{a, b}^{(i)}=z_{a}^{i} z_{b}^{n-l}$ for $i=0, \ldots, n$. Hence

$$
\left|f_{a, b}^{(i)}\right|=a i+b(q-2-i) \equiv(a+b) i-2 b(i+1) \equiv-j i-2 b(i+1) \equiv j .
$$

Hence we have $(2 b+j)(i+1) \equiv 0$. Since $i \neq q-1$, we have $j \equiv-2 b$. Since $a+b \equiv-j \equiv 2 b$, we have $a=b$, which is a contradiction. Therefore $y_{a, b}$ is a base point of $\left|K_{M}+n D+j N\right|$.

Conversely, let $x=\left(x_{0}: \cdots: x_{q-1}\right)$ be a base point of $\left|K_{M}+n D+j N\right|$. Let $t \equiv-j / 2$. Since $f=z_{t}^{n} \in S_{n}^{j}$, we have $x_{t}=0$ at $x$. Suppose that $x_{a} \neq 0$ for $a \neq t$. Consider the form $f=z_{a}^{l} z_{b}^{n-l}$ where $b \neq a$ and $i=0, \ldots, n$. Since $n=q-2, f \in S_{n}^{J}$ if and only if $(a-b) i-2 b \equiv j$. For any $a, b, j$ with $a \neq b$, this relation gives a unique $i \in Z / q Z$. Moreover $i \equiv q-1$ only if $a+b \equiv-j$. Hence, if $a+b+j \not \equiv 0$, we have $z_{a}^{l} z_{b}^{n-l} \in S_{n}^{J}$ for some $i \leq n$. Since $x$ is a base point and $x_{a} \neq 0$, we have $x_{b} \neq 0$ for $b \not \equiv-a-j$. This shows that the base points of $\left|K_{M}+n D+j N\right|$ are exactly the points $y_{a, b}$ with $a+b+j \equiv 0$.

(2) We assume that $d \geq 1$ (the case of $d=0$ is (1)). As in (1), we may regard $S_{n+d}^{J}$ as the basis of $H^{0}((n+d) D+j N)$.

Take $a, b$ and $c \equiv(a+b+j) / d$. If $c \equiv a$ or $c \equiv b$, we can replace $(a, b)$ by $(a+1, b-1)$ or $(a-1, b+1)$ so that $a, b, c$ are mutually distinct in $\boldsymbol{F}_{q}$, because $q \geq 5$.

Let $L_{c}=X \cap\left(\bigcap_{i \neq a, b, c}\left(z_{l}=0\right)\right)$ and let $Z=(d+1) y_{a, b}$ be a curvilinear zero cycle along $\pi\left(L_{c}\right)$. The image of the map $\rho: H^{0}(M,(n+d) D+j N) \rightarrow$ $H^{0}(Z,(n+d) D+j N)$ is generated by monomials $h$ in $S_{n+d}^{J}$ such that $h\left(L_{c}\right) \neq 0$. They are of the form $h=z_{c}^{k} z_{a}^{l} z_{b}^{n+d-l-k}$ with $k \leq d$. In case 
$k=d$, we have $i \leq n=q-2$ and $i+1 \not \equiv 0$. But $j \equiv c d+a i-(2+i) b$ yields $(a-b)(i+1) \equiv 0$ since $c \equiv(a+b+j) / d$. This contradicts $a \neq b$, so we conclude $k<d$.

Next, recall that $\left.F\right|_{L_{c}}$ is of the form $z_{a}^{q}+z_{b}^{q}+z_{c}^{q}+$ (other terms). Hence $\left.z_{a}^{q}\right|_{L_{c}}$ can be expressed by terms of smaller degrees in $z_{a}$. Thus we may assume that $g$ in of the form $z_{c}^{k} z_{a}^{l} z_{b}^{n+d-l-k}$ with $k<d$ and $0 \leq i<q$. Since $j \equiv k c+a i+$ $b(n+d-i-k) \equiv(a-b) i+k(c-b)+b(n+d), \quad i$ is uniquely determined for each $k=0, \ldots, d-1$. Therefore $\operatorname{Im}(\rho)$ is generated by $d$ monomials, so $\operatorname{dim} \operatorname{Im}(\rho) \leq d$, hence $\rho$ is not surjective, and $K_{M}+(n+d) D+j N$ is not $d$-spanned.

We get the following corollary by setting $j=0$ :

COROLlaRY. $\left|K_{M}+n D\right|$ has finitely many base points and $K_{M}+(n+d) D$ is not $d$-spanned for $0 \leq d \leq q-1$.

\section{General case}

By taking a divisor $D_{i}$ in the preceding example, we will get examples of dimension $q-3$. This process gives examples of any dimension.

Let $X^{\prime}$ be a smooth hypersurface in $\boldsymbol{P}^{n+1}$ defined by a $G$-invariant homogeneous polynomial of degree $q$ as in section 2 , and set $M^{\prime}=X^{\prime} / G$, where the $G$-action is given by $g\left(z_{0}^{\prime}: \cdots: z_{n+1}^{\prime}\right)=\left(g^{w_{0}} z_{0}^{\prime}: \cdots: g^{w_{n+1}} z_{n+1}^{\prime}\right)$ for some $w_{0}, \ldots, w_{n+1}$ with $0 \leq w_{0}<w_{1}<\cdots<w_{n+1} \leq q-1$. Let $T=\{0, \cdots, q-1\} \backslash$ $\left\{w_{0}, \ldots, w_{n+1}\right\}$ and $s=\# T=q-2-n$. Then there are a linear embedding $\imath: \boldsymbol{P}^{n+1} \subset \boldsymbol{P}^{q-1}$ and a smooth hypersurface $X$ in $\boldsymbol{P}^{q-1}=\left\{\left(z_{0}: \cdots: z_{q-1}\right)\right\}$ defined by a $G$-invariant homogeneous polynomial of degree $q$ such that $X^{\prime}=X \cap \boldsymbol{P}^{n+1}$, where the $G$-action on $\boldsymbol{P}^{q-1}$ is given by $g: z_{j} \mapsto g^{J} z_{j}$ and $z_{j}^{\prime}=\imath^{*} z_{w_{j}}$. The pair $X$ and $M=X / G$ is a fundamental one as in section 3. Moreover $M^{\prime}$ is identified with the submanifold $\bigcap_{i \in T} D_{i}$ in $M$. Set $D^{\prime}=\left.D_{w_{0}}\right|_{M^{\prime}}$ and $N^{\prime}=\left.N\right|_{M^{\prime}} \in \operatorname{Pic}\left(M^{\prime}\right)$. Then

$$
\begin{aligned}
K_{M^{\prime}}+t D^{\prime}+j N^{\prime} & =\left.\left(K_{M}+(s+t) D_{w_{0}}+\left(\sum_{\imath \in T}\left(i-w_{0}\right)+j\right) N\right)\right|_{M^{\prime}} \\
& =\left.\left(K_{M}+(s+t) D+\left(\sum_{t \in T} i+t w_{0}+j\right) N\right)\right|_{M^{\prime}}
\end{aligned}
$$

by the adjunction formula.

Since $H$ is an ample divisor on $X$, we have

$$
0=H^{1}\left(X, \mathcal{O}_{X}(-s H)\right) \cong H^{1}\left(M, \pi_{*} \mathcal{O}_{X}(-s H)\right)=\oplus_{J} H^{1}\left(M, \mathcal{O}_{M}(-s D+j N)\right) .
$$

Hence we have $H^{1}\left(M, \mathcal{O}_{M}(-s D+j N)\right)=0$ for any $j$. Therefore the restriction map $H^{0}\left(M, K_{M}+(s+t) D+\left(\sum i+t w_{0}+j\right) N\right) \rightarrow H^{0}\left(M^{\prime}, K_{M^{\prime}}+t D^{\prime}+j N^{\prime}\right) \quad$ is 
surjective for any $t, j$. Hence $\left|K_{M^{\prime}}+t D^{\prime}+j N^{\prime}\right|$ has a base point if $\left|K_{M}+(s+t) D+j^{\prime} N\right|$ has a base point on $\bigcap_{i \in T} D_{i}$ for $j^{\prime}=\sum_{t \in T} i+t w_{0}+j$. Similarly, $K_{M^{\prime}}+t D^{\prime}+j N^{\prime}$ is not $d$-spanned if $H^{0}\left(M, K_{M}+(s+t) D+j^{\prime} N\right)$ is not $d$-spanned at a point in $\bigcap_{i \in T} D_{i}$ along curvilinear zero cycles in $M^{\prime}$.

By this observation and Theorem 3, we get the following:

THEOREM 4. Let $n \geq 2$ and let $M^{\prime}$ be as before. For $a \neq b$, let $y_{a, b}$ be the point on $M^{\prime}$ defined by $z_{i}^{\prime}=0$ for all $i \neq a, b$. Let $j^{\prime}=\sum_{l \in T} i+(n+d) w_{0}+j$. Then

(1) If there exist $a, b$ such that $w_{a}+w_{b}+j^{\prime} \equiv 0$ and that $a \neq b$ then $y_{a, b}$ is $a$ base point of $\left|K_{M^{\prime}}+n D^{\prime}+j N^{\prime}\right|$.

(2) If there exists $w_{c}$ such that $w_{c} \equiv\left(w_{a}+w_{b}+j^{\prime}\right) / d$ and that $c$ is different from $a, b$ then $K_{M^{\prime}}+(n+d) D^{\prime}+j N^{\prime}$ is not d-spanned for $d \leq q-1$.

Proof. If $q=n+2$, this is just Theorem 3. Let $q>n+2$.

(1) By Theorem 3, $y_{a, b}$ is a base point of $\left|K_{M}+(q-2) D+j^{\prime} N\right|$ if $w_{a}+w_{b}+j^{\prime} \equiv 0$. Hence the above observation applies.

(2) By Theorem 3, $\left|K_{M}+(q-2+d) D+j^{\prime} N\right|$ is not $d$-spanned along the curvilinear zero cycle $Z$ over $y_{a, b}$ along $L_{c}$. Hence the above observation applies.

If $n=q-2$ then the canonical sheaf of $M$ is trivial and $M$ is of Kodaira dimension 0 . If $n<q-2$, the canonical sheaf of $M$ is ample and $M$ is of general type.

Now we give explicit examples by applying Theorem 4 .

(1) Consider first the case where $n \geq 3$ and $d \geq 0$. Choose a prime number $q$ such that $q \neq p$ and that $q \geq \max (n+3, d+1)$.

Assume that $q-2-n=2 m$ is even and set

$$
T=\left\{\frac{q-1}{2}-(m-1), \frac{q-1}{2}-(m-2), \ldots, \frac{q-1}{2}+m\right\} .
$$

Then we have $\sum_{l \in T} i=0$ and $\{0,1,2, q-2, q-1\} \subset\left\{w_{0}, \ldots, w_{n+1}\right\}$. Let $D^{\prime}=\left.D_{0}\right|_{M^{\prime}}$. In this case, we have $j^{\prime} \equiv 0$. Let $\{a, b\}=\{1, q-1\}$. Then we have $a+b+j^{\prime} \equiv 0$ and $c \equiv\left(a+b+j^{\prime}\right) / d \equiv 0$. Hence $K_{M^{\prime}}+n D^{\prime}$ has a base point $y_{1, q-1}$ and $K_{M^{\prime}}+(n+d) D^{\prime}$ is not $d$-spanned along $L_{0}$ at $y_{1, q-1}$.

Assume next that $q-2-n=2 m+1$ is odd. Note that $(q-1) / 2+m<$ $q-3$. Indeed, $2(q-3)-(q-1)-2 m=q-2 m-5=n-2>0$. Let

$$
T=\left\{\frac{q-1}{2}-(m-1), \frac{q-1}{2}-(m-2), \ldots, \frac{q-1}{2}+m, q-3\right\} .
$$

Then we have $\sum_{l \in T} i=-3$ and $\{0,1,2, q-2, q-1\} \subset\left\{w_{0}, \ldots, w_{n+1}\right\}$. Let $D^{\prime}=\left.D_{0}\right|_{M^{\prime}}$. In this case, we have $j^{\prime} \equiv-3$. Let $\{a, b\}=\{1,2\}$. Then we have $a+b+j^{\prime} \equiv 0$ and $c \equiv\left(a+b+j^{\prime}\right) / d \equiv 0$. Hence $K_{M^{\prime}}+n D^{\prime}$ has a base point $y_{1,2}$ and $K_{M^{\prime}}+(n+d) D^{\prime}$ is not $d$-spanned along $L_{0}$ at $y_{1,2}$. 
(2) Consider next the case where $n=2$ and $d \geq 0$. Choose a prime number $q$ with $q \geq \max (n+3=5, d+3)$ and set $c \equiv-1 /(d+1) \in Z / q Z$. Since $q \geq d+3$, we have $c \neq 0,1$. Let $a=0$ and $b$ be different from $1, a, c$. Define the ambient space $\boldsymbol{P}^{n+1}$ of $X$ to be $\bigcap_{i \neq 1, a, b, c}\left(x_{l}=0\right)$ in $\boldsymbol{P}^{q-1}$. Then $\left(x_{a}: x_{1}: x_{b}: x_{c}\right)$ gives $i \neq 1, a, b, c$ homogeneous coordinater of $\boldsymbol{P}^{n+1}$. Let $D^{\prime}=\left.D_{0}\right|_{M^{\prime}}$. In this case, we have $j^{\prime} \equiv-(1+a+b+c)$. If $d=0$ then $a+b+j^{\prime} \equiv-(c+1) \equiv 0$. Hence $K_{M^{\prime}}+n D^{\prime}$ has a base point $y_{a, b}$. If $d>0$ then $\left(a+b+j^{\prime}\right) / d \equiv-(1+c) / d \equiv c$. Hence $K_{M^{\prime}}+(n+d) D^{\prime}$ is not $d$-spanned along $L_{c}$ at $y_{a, b}$.

Thus we have the examples of $n \geq 2$ for any $d \geq 0$ and $p \geq 0$.

\section{REFERENCES}

[BB] E. Ballico AND M. Beltrametti, On the $k$-spannedness of the adjoint line bundle, Manuscripta Math., 76 (1992), 407-420.

[BFS] M. Beltrametti, P. Francia and A. J. Sommese, On Reider's method and higher order embeddings, Duke Math. J., 58 (1989), 425-439.

[EL] L. EIN AND R. LAZARSFELD, Global generation of puricanonical and adjoint linear series on projective threefolds, J. Amer. Math. Soc., 6 (1993), 875-903.

[F1] T. FustTA, Classification Theories of Polarized Varieties, London Math. Soc. Lecture Notes Ser., 155, Cambridge Unıv. Press, 1990.

[F2] T. FuנITA, Remarks on Ein-Lazarsfeld criterion of spannedness of adjoint bundles of polarized threefold, preprint.

[K] Y. Kawamata, On Fujita's freeness conjecture for 3-folds and 4-folds, preprint (1995).

[R] I. ReIDER, Vector bundles of rank 2 and linear systems on algebraic surfaces, Ann. of Math., 127 (1998), 309-316.

DePaRTMENT OF Mathematics

TOKYo INSTITUTE OF TECHNOLOGY

2-12-1 OH-OKayama, Meguro-KU

TOKYO 152-8551, JAPAN

Telefax: +81-3-5734-2738

E-mail: kawachı@math.titech.ac.jp 